\title{
Salpingectomia Profilática como Método de Contraceção Definitiva: Estudo Transversal em Portugal
}

\author{
Opportunistic Salpingectomy for Permanent \\ Contraception: A Cross Sectional Study in Portugal
}

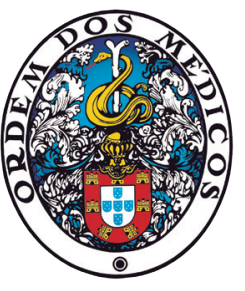

\author{
Verónica SÃO PEDRO $\rrbracket^{1}$, Rafaela PIRES ${ }^{1}$, Fernanda SANTOS ${ }^{1}$, Carla TOVIM RODRIGUES ${ }^{1}$, Isabel SANTOS SILVA ${ }^{1}$, \\ Maria do Céu ALMEIDA' ${ }^{1}$, Fernanda ÁGUAS ${ }^{1}$ \\ Acta Med Port 2021 Apr;34(4):258-265 - https://doi.org/10.20344/amp.14033
}

\section{RESUMO}

Introdução: A salpingectomia profilática foi proposta como estratégia de redução do risco de cancro do ovário e método de contraceção definitiva. O objetivo deste estudo foi conhecer os procedimentos realizados a nível nacional para contraceção definitiva de intervalo e peri-parto, a opinião e motivações dos clínicos, e os fatores demográficos ou profissionais influentes.

Material e Métodos: Este é um estudo analítico transversal, baseado num questionário original enviado durante o ano de 2019 a especialistas e internos de Ginecologia-Obstetrícia a exercer em Portugal.

Resultados: Obtivemos 225 respostas provenientes de médicos a exercer em 42 hospitais (37 públicos). A laqueação tubar laparoscópica por eletrocoagulação e corte $(61 \%)$ foi o método mais frequentemente utilizado na mulher não grávida, seguido da salpingectomia (28\%). Os principais motivos apontados para não realizar salpingectomia foram o aumento do tempo operatório $(48,5 \%)$ e tratar-se de procedimento não equacionado (45,5\%). Em alguns hospitais, a realização deste método dependia da decisão da equipa cirúrgica. No contexto per-cesariana, a técnica mais comum foi a de Pomeroy modificada (54\%), seguida da salpingectomia (32,5\%), com uma concentração no Norte do país com significado estatístico. A maioria (69\%) dos participantes consideraram que a salpingectomia deveria ser o procedimento disponibilizado.

Discussão: Apesar da evidência científica ainda ser escassa, a salpingectomia per-cesariana parece exequível e segura, podendo representar a melhor oportunidade para intervenção no contexto da contraceção definitiva.

Conclusão: A salpingectomia profilática não é o procedimento de esterilização mais comum em Portugal, mas foi considerada como a escolha mais adequada. Os seus benefícios e riscos devem ser discutidos com as mulheres.

Palavras-chave: Esterilização Reprodutiva; Inquéritos e Questionários; Neoplasias dos Ovários/prevenção e controlo; Procedimentos Cirúrgicos Profiláticos; Salpingectomia

\section{ABSTRACT}

Introduction: Opportunistic bilateral salpingectomy has been proposed as an ovarian cancer risk-reducing strategy namely as a means of tubal sterilization. We aimed to assess what were the procedures for interval and peripartum sterilization carried out nationwide, related motivational aspects and influential demographic or professional factors.

Material and Methods: Cross-sectional study based on an original survey sent to Obstetrics and Gynecology specialists and residents from across the country in 2019.

Results: Two hundred and twenty-five answers were obtained from 42 institutions (37 from the public sector). Laparoscopic tubal electrocoagulation (61\%) was the most common procedure for interval sterilization followed by salpingectomy (28\%). Major reasons pointed out for not performing salpingectomy were increased operative time (48.5\%) and procedure not considered (45.5\%). In some hospitals, the choice of salpingectomy depended on specific criteria namely surgical team decision. During cesarean-section, sterilization was most frequently performed using the modified Pomeroy technique (54\%), followed by salpingectomy (32.5\%), with a statistically significant prevalence in the north of the country. Sixty-nine percent of Portuguese Obstetrics and Gynecology residents and specialists consider that salpingectomy should be the procedure offered to women asking for definitive contraception.

Discussion: Although data are limited, salpingectomy at the time of cesarean delivery appears feasible and safe and this context might represent the best opportunity for intervention.

Conclusion: Opportunistic salpingectomy is not the most common sterilization procedure performed in Portugal, but it was considered the best choice to offer. Its benefits and risks should be discussed with women.

Keywords: Ovarian Neoplasms/prevention \& control; Prophylactic Surgical Procedures; Salpingectomy; Sterilization, Reproductive; Surveys and Questionnaires

\section{INTRODUÇÃO}

O cancro do ovário é o cancro ginecológico mais letal nos países desenvolvidos e a sua incidência tem aumentado nas últimas décadas, sendo responsável em 2018 por 184799 mortes a nível mundial. ${ }^{1}$ As estratégias de prevenção secundária propostas até à data, nomeadamente com cancer antigen 125 (CA 125) e ecografia ginecológica, não se mostraram eficazes, levando a intervenções e compli- cações desnecessárias sem uma diminuição da mortalidade $^{2-5} \mathrm{O}$ carcinoma seroso de alto grau (CSAG), subtipo histológico mais comum, é diagnosticado em estádios avançados (estádios III e IV, segundo a classificação da Federação Internacional de Ginecologia-Obstetrícia), em mais de $70 \%$ dos casos, e apesar da evolução dos tratamentos de quimioterapia nos últimos 30 anos, a sobrevida

\footnotetext{
1. Serviço de Ginecologia e Obstetrícia B. Centro Hospitalar e Universitário de Coimbra. Coimbra. Portugal.

$\triangle$ Autor correspondente: Verónica São Pedro. veronicasaopedro@gmail.com

Recebido: 03 de maio de 2020 - Aceite: 01 de julho de 2020 - Online issue published: 01 de abril de 2021
}

Copyright $\odot$ Ordem dos Médicos 2021 
aos cinco anos mantém-se em apenas 35\%.,

Estratégias de prevenção primária afiguram-se relevantes e neste sentido está preconizada a anexectomia profilática aos 35 anos ou após desejo reprodutivo completo em mulheres com mutação conhecida no breast cancer gene 1 (BRCA1 - risco até $60 \%$ de CSAG ao longo da vida), ou numa idade mais tardia se a mutação diagnosticada estiver associada a um risco inferior. ${ }^{8} \mathrm{Da}$ análise histológica sistematizada e extensa das trompas destas mulheres verificou-se a presença de serous tubal intraepitelial carcinomas (STIC) e carcinomas ocultos em percentagem elevada de doentes. ${ }^{9}$ Isto permitiu identificar uma sequência de carcinogénese na qual os STIC constituem as lesões precursoras, que se destacam e implantam no ovário, dando origem ao CSAG do ovário, trompa e peritoneu. ${ }^{6}$ Estudos subsequentes confirmaram que os perfis de expressão génica e imunohistoquímica do CSAG se assemelham mais ao epitélio mülleriano (das trompas) do que ao epitélio mesotelial (de superfície do ovário).9,10

Este novo modelo de carcinogénese, com origem tubar, tem implicações clínicas importantes nomeadamente no que diz respeito à prevenção primária, uma vez que a maioria dos casos de CSAG do ovário (90\% - 95\%) surge nas mulheres de baixo risco. Assim, com o objetivo de reduzir a incidência do CSAG do ovário, trompa e peritoneu, - British Columbia's Ovarian Cancer Research Program (grupo OvCaRe) propôs em 2010 a realização de salpingectomia profilática, ou seja, a exérese tubar em mulheres saudáveis no contexto de uma cirurgia pélvica por outra indicação. Serão necessários mais alguns anos para avaliar o impacto real da remoção das trompas na incidência e mortalidade do cancro do ovário, mas a evidência atual demonstra uma ausência de riscos (nomeadamente cirúrgi$\cos ^{6}$ e de diminuição da reserva ovárica) ${ }^{11}$ e uma análise de custo-eficácia favorável. ${ }^{12}$ Por este motivo, as organizações internacionais, nomeadamente o American College of Obstetricians and Gynecologists (ACOG) e o Royal College of Obstetricians and Gynaecologists (RCOG), já recomendam a salpingectomia adicionalmente à histerectomia por patologia benigna, ou como alternativa à laqueação tubar. ${ }^{13,14}$ Está ainda em estudo a exequibilidade da salpingectomia profilática no contexto peri-parto ${ }^{15-19}$ ou de outras cirurgias abdominais não ginecológicas (nomeadamente colecistectomia laparoscópica), e como primeira estratégia redutora de risco em mulheres com mutação $B R C A 1 / 2$, o que permitiria adiar a ooforectomia e reduzir os riscos de osteoporose e doença cardiovascular associados a uma falência prematura da função ovárica. ${ }^{20}$

Os benefícios adicionais da salpingectomia profilática são a diminuição do risco de reintervenção cirúrgica por dor pélvica crónica, hidrossalpinge, piossalpinge, abcesso tubo-ovárico e gravidez ectópica, e a sua superior eficácia contracetiva. ${ }^{21} \mathrm{Em}$ contrapartida, em caso de arrependimento e desejo de maternidade posterior, é impossível uma recuperação cirúrgica da função tubar, restando apenas o recurso a técnicas de procriação medicamente assistida.

Em Portugal, a salpingectomia profilática passou a ser contemplada em 2020, no Consenso sobre Contraceção ${ }^{22}$ como procedimento de contraceção definitiva, e no Consenso de Cancro Ginecológico ${ }^{23}$ no contexto da histerectomia com preservação ovárica ou outra cirurgia intra-abdominal. A distribuição nacional dos diferentes procedimentos de esterilização feminina na mulher não grávida e no contexto peri-parto não é conhecida, podendo variar amplamente entre e dentro dos serviços de acordo com os protocolos existentes, as crenças individuais, e o conhecimento da evidência disponível. Atendendo ao exposto, desenhámos o primeiro estudo português com o objetivo de retratar a referida distribuição e dar a conhecer as motivações subjacentes às práticas. Secundariamente, procurámos identificar fatores demográficos e profissionais na origem das opiniões discrepantes e eventuais barreiras à instituição da salpingectomia.

\section{MATERIAL E MÉTODOS}

Foi desenhado um estudo analítico transversal, cujos dados foram obtidos através da aplicação de um questionário original de 28 perguntas (ver Apêndice 1: https://www. actamedicaportuguesa.com/revista/index.php/amp/article/ view/14033/Apendice_01.pdf) a uma amostra de internos e especialistas em Ginecologia-Obstetrícia, a exercer em Portugal continental e regiões autónomas. Foi realizado online através da plataforma Google Forms e esteve disponível de 10 de agosto de 2019 a 30 de novembro de 2019 .

Previamente à sua implementação, foi testada a adequação ao objetivo do trabalho através da realização de um teste piloto por especialistas e internos de Ginecologia-Obstetrícia do nosso serviço. O questionário foi posteriormente divulgado nos diferentes serviços públicos de Ginecologia-Obstetrícia e foi difundido a nível nacional pela Portuguese Network of Trainees in Obstetrics and Gynaecology (PONTOG), pela Sociedade Portuguesa de Ginecologia (SPG) e pela rede social Facebook ${ }^{\circledR}$.

Este estudo não careceu de aprovação pela comissão de ética local dada a ausência de intervenções e de utilização de dados de doentes. O consentimento informado dos participantes foi garantido no momento da submissão da resposta já que claramente se informava nessa etapa que ao submeter o questionário estaria a consentir com a utilização dos seus dados para análise estatística. O software utilizado para esta análise foi o IBM SPSS Statistics $₫$ versão 25 (SPSS Inc., Chicago, Illinois, USA), e os resultados analisados com recurso aos testes qui-quadrado, teste exato de Fisher (comparação de grupos relativamente a variáveis nominais), Mann-Whitney, Kruskal Wallis (comparação de dois ou mais grupos relativamente a variáveis contínuas com distribuição não gaussiana) e correlação de Spearman (análise da relação entre duas variáveis ordinais e/ou contínuas com distribuição não gaussiana). Foi atribuída significância estatística a valores $p<0,05$. A análise foi efetuada por instituição pública relativamente ao procedimento preferencialmente oferecido, e por participante relativamente a todas as outras questões. 


\section{RESULTADOS}

Foram obtidas 225 respostas (ver Apêndice 2: https:// www.actamedicaportuguesa.com/revista/index.php/amp/ article/view/14033/Apendice_02.pdf) de especialistas e internos de Ginecologia-Obstetrícia de 42 instituições hospitalares: 37 hospitais públicos (213 respostas) e cinco privados. A caracterização demográfica e profissional dos participantes encontra-se descrita na Tabela 1. Estiveram representados $84 \%$ dos serviços públicos nacionais (distribuição na Fig. 1), englobando a totalidade dos serviços de tipologia A1, A2 e B1. Verificou-se uma correlação positiva entre o número de respostas e a tipologia do serviço (coeficiente de correlação $=0,432$; valor $p=0,008$ ), sendo que serviços maiores proporcionaram um número de respostas superior.

A técnica cirúrgica preferencialmente oferecida às muIheres que pretendem contraceção definitiva foi a laqueação tubar laparoscópica com eletrocoagulação e corte em
$61 \%$ dos hospitais, seguida da salpingectomia laparoscópica em $28 \%$ e da laqueação tubar laparoscópica com anéis de Yoon, laqueação tubar por laparotomia e salpingectomia por laparotomia com menor representação (Fig. 1). Não se verificaram entre os serviços respondentes diferenças estatisticamente significativas na realização de salpingectomia no que diz respeito aos critérios região, tipologia, idoneidade formativa e disponibilização de cirurgia de repermeabilização tubar.

Questionados sobre a possibilidade de a salpingectomia ser uma opção considerada, $47 \%$ dos participantes referiram que podia ser uma opção cirúrgica a oferecer no seu hospital, dependendo de critérios específicos (Tabela 2), dos quais se destacou a equipa cirúrgica presente (65\%). É de realçar que a preferência da mulher foi o critério mais identificado na alínea 'outras opções'. A maioria dos inquiridos (84\%) admitiu desconhecer a percentagem de salpingectomia versus laqueação no seu hospital.

Tabela 1 - Caracterização da amostra

\begin{tabular}{|c|c|c|c|c|}
\hline Participantes (225) & $\mathbf{n}(\%)$ & Serviços públicos & $\mathrm{n}(\%)^{*}$ & $\mathbf{n}(\%)^{\dagger}$ \\
\hline Idade & & Região & & \\
\hline $25-30$ & $44(19,6)$ & Norte & $12(32,4)$ & $58(27,2)$ \\
\hline $30-35$ & $58(25,8)$ & Centro & $6(16,2)$ & $64(30,0)$ \\
\hline $35-40$ & $43(19,1)$ & Lisboa e Vale do Tejo & $13(35,1)$ & $70(32,9)$ \\
\hline $40-45$ & $18(8,0)$ & Sul (Alentejo e Algarve) & $4(10,8)$ & $12(5,6)$ \\
\hline $45-50$ & $7(3,1)$ & Regiões autónomas & $2(5,4)$ & $9(4,2)$ \\
\hline $50-55$ & $14(6,2)$ & & & \\
\hline $55-60$ & $24(10,7)$ & Tipologia & & \\
\hline $60-65$ & $15(6,7)$ & A1 & $5(13,5)$ & $68(31,9)$ \\
\hline \multirow[t]{2}{*}{$65-70$} & $2(0,9)$ & A2 & $8(21,6)$ & $46(21,6)$ \\
\hline & & B1 & $16(43,2)$ & $65(30,5)$ \\
\hline Género & & B2 & $8(21,6)$ & $34(16,0)$ \\
\hline Feminino & $180(80,0)$ & & & \\
\hline \multirow[t]{2}{*}{ Masculino } & $45(20,0)$ & \multicolumn{3}{|l|}{ Com idoneidade formativa } \\
\hline & & Sim & $32(86,5)$ & $197(92,5)$ \\
\hline Experiência & & Não & $5(13,5)$ & $16(7,5)$ \\
\hline Interno & $68(30,2)$ & & & \\
\hline \multirow[t]{2}{*}{ Especialista } & $157(69,8)$ & \multicolumn{3}{|c|}{ Disponibiliza cirurgia repermeabilização tubar } \\
\hline & & Sim & $5(13,5)$ & $52(24,4)$ \\
\hline Realiza consulta de PF? & & Não & $32(86,5)$ & $161(75,6)$ \\
\hline Sim & $127(56,4)$ & & & \\
\hline Não & $98(43,6)$ & & & \\
\hline
\end{tabular}

\begin{tabular}{lc}
\hline Realiza cirurgia de CD ${ }^{\ddagger}$ & \\
\hline Sim & $166(73,8)$ \\
Não & $59(26,2)$
\end{tabular}

\begin{tabular}{lc}
\hline Realiza cesarianas? \\
\hline Sim & $211(93,8)$ \\
Não & $14(6,2)$
\end{tabular}

${ }^{*}$ Por total de serviços públicos -37 ; ${ }^{\dagger}$ Por total de participantes $-213 ;{ }^{\ddagger}$ CD - Contraceção definitiva 
A

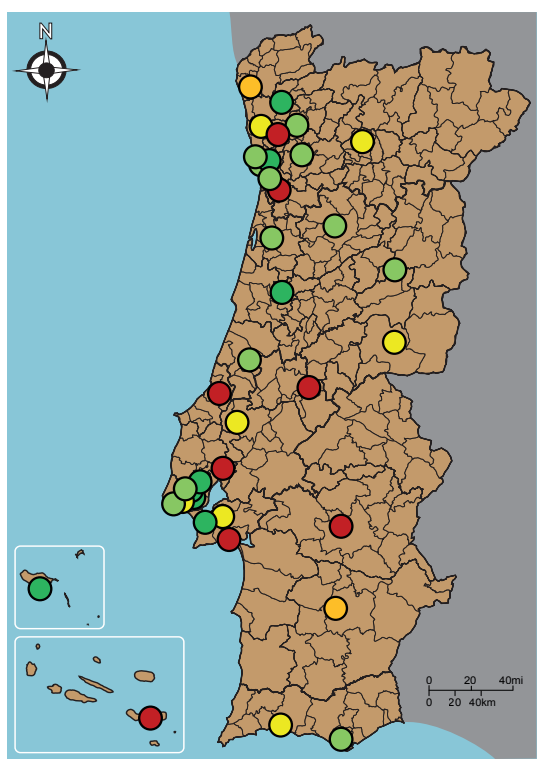

1 resposta

2 respostas

3 - 4 respostas

$5-7$ respostas

$\geq 8$ respostas
B

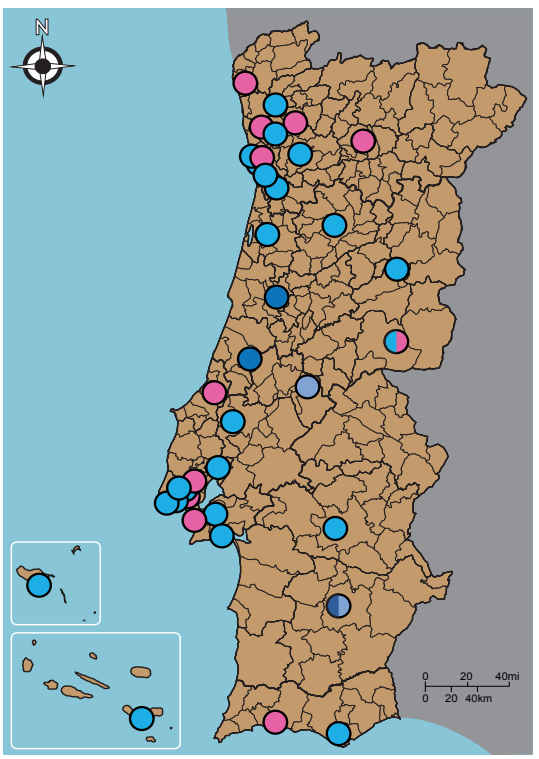

Laqueação tubar laparoscópica (eletrocoagulação e corte)

Laqueação tubar laparoscópica (anéis deYoon)

Salpingectomia por laparoscopia

Laqueação tubar por laparoscopia

Salpingectomia por laparoscopia

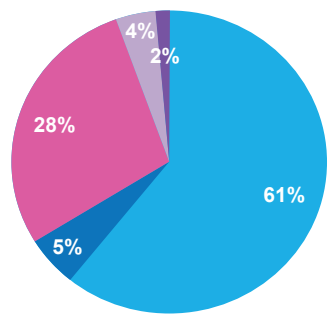

C

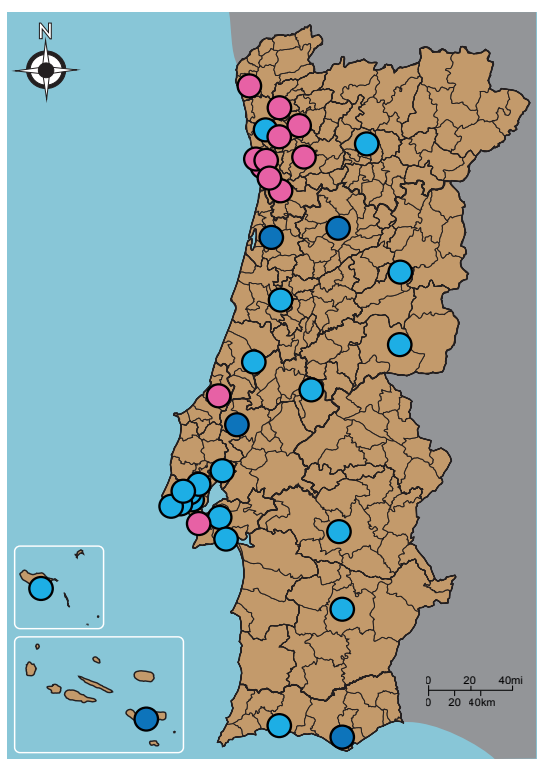

Pomeroy modificada

Parkland

Salpingectomia

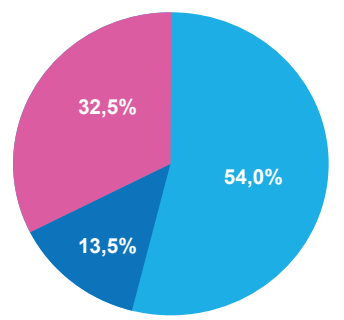

Figura 1 - Distribuição por instituição do número de respostas $(A)$, do procedimento oferecido à mulher não grávida $(B)$ e per-cesariana (C)

Tabela 2 - Critérios para salpingectomia profilática quando não protocolada

\begin{tabular}{lc}
\hline Critérios utilizados* & $\mathbf{n}(\%)$ \\
\hline Idade superior a 35 anos & $8(7,5)$ \\
Patologia associada contraindicando gravidez & $24(22,6)$ \\
Caso social & $12(11,3)$ \\
Três ou mais filhos & $9(8,5)$ \\
Antecedentes familiares de carcinoma do ovário & $41(38,7)$ \\
Mutação BRCA conhecida adiando a ooforectomia para mais tarde & $33(31,1)$ \\
Depende mais da equipa cirúrgica do que de algum critério específico & $69(65,1)$ \\
Outros (acrescentados pelos participantes): & $11(10,4)$ \\
Preferência da utente & 6 \\
Proposta cirúrgica & 2 \\
Contexto per-cesariana & 2 \\
Patologia ginecológica associada & 1 \\
\hline
\end{tabular}

só responderam a esta questão os participantes (105) que referiram que no seu hospital a salpingectomia podia ser equacionada perante critérios específicos - podiam selecionar mais do que uma opção 
Apenas $15 \%$ dos participantes referiram que a salpingectomia não fazia parte das opções cirúrgicas oferecidas no seu hospital e apontaram maioritariamente como motivos (Tabela 3) o aumento do tempo cirúrgico (49\%) e alternativa não equacionada (46\%).

Relativamente à transmissão de informação às mulheres com desejo de contraceção definitiva, apenas 37\% dos participantes consideraram que no seu hospital era disponibilizada sistematicamente informação sobre as vantagens e desvantagens da salpingectomia versus a laqueação, sendo oferecida hipótese de escolha entre estes procedimentos. Esta percentagem decresceu para $31 \%$ no contexto da gravidez. A opção pretendida, salpingectomia ou laqueação, foi referida por $34,5 \%$ dos participantes como constando do consentimento informado. Verificou-se uma diferença estatisticamente significativa na disponibilização sistemática da informação acima referida entre os hospitais onde se realizava salpingectomia versus aqueles onde se realizava laqueação: em $78,1 \%$ vs $22,7 \%$ a mulher não grávida é informada (valor $p<0,001$ ) e em $53,2 \%$ vs $29,3 \%$ a grávida é informada (valor $p=0,012$ ).

No contexto per-cesariana todos os participantes consideraram que no seu hospital se efetuava contraceção definitiva por opção da mulher, quando previamente requerida durante a gravidez, com uma frequência globalmente estimada entre $5 \%$ e $10 \%$. A técnica cirúrgica preferencialmente oferecida (Fig. 1) foi a de Pomeroy modificada em 54\% dos hospitais, seguida da salpingectomia em $32,5 \%$ e da técnica de Parkland em 13,5\%. Verificou-se uma diferença estatisticamente significativa na realização de salpingectomia per-cesariana entre a região norte e o resto do país $(83 \%$ vs $8 \%$, valor $p<0,001)$.

Avaliando diretamente se a salpingectomia seria uma opção considerada no contexto per-cesariana, $46 \%$ dos participantes consideraram que dependia da equipa cirúrgica e $31 \%$ referiram que não era uma opção na sua instituição, tendo apontado como principais motivos de tal situação (Tabela 3) tratar-se de alternativa não equacionada $(57 \%)$, o aumento do tempo cirúrgico $(42 \%)$ e o medo de complicações (36\%). Dos 141 participantes que assumiram a realização de salpingectomia per cesariana no seu hospital, $97 \%$ referiram não ter conhecimento de nenhuma complicação cirúrgica diretamente atribuída a este procedimento. As complicações referidas pelos restantes foram hemorragia não especificada (dois casos), hemorragia intraoperatória com necessidade de histerectomia per-cesariana (um caso) e hemoperitoneu (um caso).

Nas situações em que a grávida expressara previamente o desejo de contraceção definitiva, mas nas quais o parto viria a ocorrer por via vaginal, apenas dois hospitais públicos da região de Lisboa e Vale do Tejo ofereciam a possibilidade de contraceção definitiva por mini-laparotomia umbilical no mesmo internamento, sendo que, num se realizava salpingectomia e no outro laqueação tubar pela técnica de Pomeroy modificada.

$\mathrm{Na}$ opinião pessoal de $69 \%$ dos participantes, o procedimento de contraceção definitiva a oferecer deveria ser a salpingectomia. A laqueação tubar foi eleita por $15 \%$ dos participantes e $10 \%$ referiram não ter opinião formada. Doze dos inquiridos preferiram especificar opções alternativas tais como informação da mulher e decisão partilhada (6), avaliação do contexto clínico (2), colocação de dispositivo intrauterino libertador de levonorgestrel (2), necessidade de mais estudos (1) e vasectomia (1). Não houve diferenças estatisticamente significativas entre os participantes que optariam por oferecer a salpingectomia versus a laqueação

Tabela 3 - Motivos para não realização de salpingectomia profilática

\begin{tabular}{lc}
\hline Motivos na mulher não grávida* & $\mathbf{n}(\%)$ \\
\hline Alternativa não equacionada & $15(45,5)$ \\
Ausência de vantagens relativamente à laqueação & $3(9,1)$ \\
Irreversibilidade do procedimento (impossibilidade de cirurgia de repermeabilização tubar) & $4(12,1)$ \\
Ausência de treino cirúrgico laparoscópico para salpingectomia & $4(12,1)$ \\
Receio de menopausa precoce & $1(3,0)$ \\
Aumento do tempo cirúrgico & $16(48,5)$ \\
A salpingectomia implica uma abordagem mais invasiva & $10(30,3)$ \\
Outros (não pretendem atualização) & $1(3,0)$ \\
\hline Motivos no contexto per-cesariana ${ }^{\dagger}$ & $\mathbf{n}(\%)$ \\
\hline Ausência de vantagens relativamente à laqueação & $4(5,8)$ \\
Irreversibilidade do procedimento & $7(10,1)$ \\
Ausência de treino cirúrgico para o procedimento & $6(8,7)$ \\
Medo de complicações & $25(36,2)$ \\
Aumento do tempo cirúrgico & $29(42,0)$ \\
Alternativa não equacionada & $39(56,5)$ \\
Outros (equipa cirúrgica) & $1(1,4)$ \\
\hline
\end{tabular}

* Só responderam a esta questão os participantes (33) que referiram que a salpingectomia não fazia parte das opções cirúrgicas oferecidas no seu hospital - podiam selecionar mais do que um motivo; † Só responderam a esta questão os participantes (69) que referiram que a salpingectomia per cesariana não fazia parte das opções cirúrgicas oferecidas no seu hospital - podiam selecionar mais do que um motivo. 
relativamente ao género, experiência (interno versus especialista), realização de consulta de planeamento familiar, cirurgia de contraceção definitiva ou cesarianas, região do país, tipologia do serviço, idoneidade formativa do serviço ou possibilidade de repermeabilização tubar. Relativamente à idade, verificou-se uma distribuição etária mais jovem dos participantes que elegeram a salpingectomia $(40,4 \pm$ 11) versus a dos participantes que elegeram a laqueação $(45,7 \pm 12,3)$ (valor $p=0,038)$.

\section{DISCUSSÃO}

Apesar da existência de métodos contracetivos de longa duração com eficácia praticamente idêntica à da laqueação tubar, muitas mulheres portuguesas continuam a optar por um método cirúrgico que lhes proporcione uma contraceção definitiva. Essa escolha pode ser aproveitada para uma diminuição potencial do risco de cancro do ovário, embora não deva ser incentivada com este intuito.

Neste contexto, apresentamos o primeiro estudo efetuado em Portugal a revelar a distribuição nacional dos procedimentos de contraceção definitiva realizados, assim como as perspetivas que estão na base da sua realização. Ao contrário de inquéritos realizados noutros países, ${ }^{24-31}$ o foco deste trabalho prende-se apenas na avaliação das práticas de contraceção definitiva, não sendo abordado o contexto da histerectomia por patologia benigna, no qual a prática da salpingectomia profilática já se encontra mais amplamente difundida. Isto permitiu a construção de um questionário mais dirigido e conciso, com o objetivo de maximizar a participação.

Nos estudos que avaliaram separadamente a realização de salpingectomia profilática no contexto da contraceção definitiva foram encontradas taxas de utilização díspares entre os diferentes países, na mulher não grávida e per-cesariana: $42 \%$ e 25\% (Austrália 2015); ${ }^{29}$ 13,3\% e $12,5 \%$ (França 2016); ${ }^{26} 71 \%$ e $64 \%$ (Áustria 2016) ${ }^{27}$ e $52,8 \%$ e 36,8\% [Estados Unidos da América (EUA) 2016]. ${ }^{24}$ Apenas nos EUA foi também avaliado o contexto do pós-parto vaginal (cuja representação é significativa no continente americano), sendo de $26,4 \%$ a taxa de utilização da salpingectomia. As amplas variações encontradas poderão ser influenciadas por diversos fatores externos, entre os quais a forma como as questões foram formuladas ou a população inquirida. Por exemplo, na Áustria, onde se verificaram as taxas de utilização mais elevadas, os inquiridos foram os chefes de departamento (ausência de anonimato) e era permitida a seleção de várias opções. ${ }^{27}$ Por sua vez, as mais baixas taxas de utilização registadas em França foram relacionadas com a preferência pela oclusão tubar com Essure ${ }^{\circledR}$ (método menos invasivo, mas atualmente descontinuado). ${ }^{26} \mathrm{O}$ facto de as publicações datarem de 2015 - 2016 pode ainda implicar uma desatualização importante das taxas (nos EUA apenas 7,2\% dos clínicos haviam reportado em 2013 uma preferência pela salpingectomia como método de contraceção definitiva de intervalo). ${ }^{30}$

Somente um estudo avaliou os motivos que levavam os clínicos a realizar ou não salpingectomia no contexto específico da contraceção definitiva (EUA 2016). $\cdot{ }^{24}$ O motivo maioritariamente alegado (por $91 \%$ dos participantes) para a realização de salpingectomia foi a diminuição do risco de cancro do ovário, trompa e peritoneu, e as principais preocupações apontadas pelos que não a realizavam foram o aumento do tempo operatório (registadas por 36\%, 29\% e $31 \%$ dos participantes, consoante o contexto de intervalo, per-cesariana e pós-parto) e o risco de complicações (por $25 \%, 48 \%$ e $44 \%$ respetivamente). Não foram encontradas diferenças estatisticamente significativas nos fatores demográficos e profissionais entre os inquiridos que oferecem salpingectomia versus laqueação.

Neste estudo não foram avaliados os motivos para a realização de salpingectomia, na medida em que o foco foi a identificação de barreiras, mas poderia ter sido interessante obter essa informação. No Norte do país, a salpingectomia é o método preferencialmente usado na mulher não grávida em $42 \%$ dos hospitais, e no contexto per-cesariana em $83 \%$ dos hospitais. Esta disparidade entre contextos pode sugerir a existência de outro motivo para a realização de salpingectomia per-cesariana, para além da diminuição do risco de cancro, pois a ser esse o motivo seria de supor uma taxa igualmente elevada na mulher não grávida. É possível que a salpingectomia per-cesariana já fosse a técnica tradicionalmente utilizada no Norte do país, pela inexistência de um propósito biológico para a trompa após laqueação ou por ser considerada uma técnica ainda mais eficaz do que a laqueação.

Relativamente aos motivos para a não realização de salpingectomia, é de destacar que a resposta 'alternativa não equacionada' foi nomeada no contexto de intervalo por $45,5 \%$ dos participantes e no contexto per-cesariana por $56,5 \%$. Por sua vez, a 'ausência de vantagens relativamente à laqueação' foi selecionada por apenas $9,1 \%$ e por $5,4 \%$ dos participantes consoante o contexto, elucidando que o facto de a salpingectomia não ser equacionada não se deve a um défice de atualização dos profissionais, e que chamar a atenção para este tema poderá fazer a diferença.

No contexto da contraceção definitiva de intervalo, o aumento do tempo operatório pela realização da salpingectomia foi reportado em apenas 10 minutos,,$^{13}$ não justificando descartar a salpingectomia por este motivo. Por outro lado, a preocupação de que o ingurgitamento vascular característico do contexto peri-parto possa levar a um aumento do risco cirúrgico não é baseada na evidência. Num estudo de coorte retrospetivo envolvendo 10741 mulheres foi descrito um aumento de 10 minutos no tempo de realização da cesariana e uma perda hemática contabilizada semelhante aquando da realização de salpingectomia em lugar de laqueação. ${ }^{15}$ Resultados idênticos foram obtidos em estudos prospetivos controlados e aleatorizados. ${ }^{16,17,19}$ No nosso estudo, apenas $3 \%$ dos participantes com experiência na realização de salpingectomia peri-parto referiram ter conhecimento de complicações hemorrágicas, conhecimento esse que não levou a que alterassem a sua preferência de método para a laqueação.

Uma vez que mais de metade dos procedimentos de 
esterilização estão descritos no contexto peri-parto, ${ }^{18}$ o parto por cesariana afigura-se como a oportunidade ideal para aumentar a utilização e potencial efeito da salpingectomia profilática, aproveitando uma cirurgia realizada por indicação obstétrica nas situações em que já foi previamente expresso pela grávida um desejo de contraceção definitiva. Neste sentido, um conhecimento mais aprofundado da experiência do Norte do país poderá contribuir para uma maior segurança na difusão da salpingectomia peri-parto pelo resto do país. A esterilização no pós-parto vaginal, contexto em que a salpingectomia profilática parece ser igualmente segura, não é uma prática comum em Portugal, pelo que não constitui uma ocasião para intervenção.

Os pontos fracos deste estudo são os inerentes a um estudo baseado em inquérito, uma vez que o valor dos dados obtidos se encontra limitado por depender da perceção dos participantes. Um ponto forte é a conjugação de respostas fechadas, que permitem uma análise quantitativa, com a possibilidade de acrescentar nova informação em resposta aberta - outra opção, sempre que o participante o considerasse relevante. Isto é particularmente importante se tivermos em conta que todas as questões eram de resposta obrigatória.

Relativamente à taxa de participação, a metodologia de divulgação do questionário tornou impossível calcular com exatidão quantos médicos tiveram acesso ao mesmo. No entanto, essa metodologia permitiu obter simultaneamente uma disseminação pela maioria dos serviços públicos do país mantendo o anonimato dos participantes (por não ser dirigida aos diretores de serviço), uma distribuição demográfica aparentemente representativa da realidade nacional e um direcionamento para os profissionais que realizam consultas de planeamento familiar e cirurgias de contraceção definitiva, situação desejável por serem os profissionais que melhor conhecem a realidade dos seus serviços e participam no processo de decisão. Desta forma foi possível analisar os dados por serviço público e por participante, e obter informação sobre os procedimentos realizados nos hospitais (com uma excelente concordância entre elementos de um mesmo serviço) e sobre os procedimentos considerados mais adequados. Neste aspeto, este estudo é único, expondo duas vertentes que não se podem presumir como coincidentes. É ainda de realçar, como ponto forte, uma taxa de resposta por serviço público de $84 \%$ no pior cenário (uma vez que não é possível garantir que o questio- nário tenha efetivamente chegado aos serviços de tipologia B2, de onde não se obteve resposta).

\section{CONCLUSÃO}

Neste estudo verificou-se que a taxa de utilização da salpingectomia profiláctica em Portugal parece estar aquém do desejável. A realização de protocolos de atuação poderá ser uma forma de assegurar que a salpingectomia não deixe de ser equacionada, uma vez que os nossos profissionais parecem estar informados e recetivos à mudança.

Este estudo realça ainda a necessidade de melhorar o aconselhamento, de forma a permitir a participação da muIher ou da grávida que pretenda contraceção definitiva no processo de decisão entre a laqueação e a salpingectomia profilática, e usufruto do potencial benefício de diminuição do risco de cancro do ovário.

\section{AGRADECIMENTOS}

Os autores gostariam de agradecer aos profissionais que dentro do seu serviço se disponibilizaram a participar na fase de pré-teste contribuindo para a adequação do questionário ao objetivo do trabalho, assim como aos profissionais que permitiram a divulgação do questionário dentro dos respetivos serviços.

\section{PROTEÇÃO DE PESSOAS E ANIMAIS}

Os autores declaram que os procedimentos seguidos estavam de acordo com os regulamentos estabelecidos pelos responsáveis da Comissão de Investigação Clínica e Ética e de acordo com a Declaração de Helsínquia da Associação Médica Mundial atualizada em 2013.

\section{CONFIDENCIALIDADE DOS DADOS}

Os autores declaram ter seguido os protocolos do seu centro de trabalho acerca da publicação de dados.

\section{CONFLITOS DE INTERESSE}

Os autores declaram não ter conflitos de interesse relacionados com o presente trabalho.

\section{FONTES DE FINANCIAMENTO}

Este trabalho não recebeu qualquer tipo de suporte financeiro de nenhuma entidade no domínio público ou privado.

\section{REFERÊNCIAS}

1. Bray F, Ferlay J, Soerjomataram I, Siegel R, Torre L, Jemal A. Global cancer statistics 2018: GLOBOCAN estimates of incidence and mortality worldwide for 36 cancers in 185 countries. CA Cancer J Clin. 2018;68:394-424.

2. Kobayashi H, Yamada Y, Sado T, Sakata M, Yoshida S, Kawaguchi R, et al. A randomized study of screening for ovarian cancer: a multicenter study in Japan. Int J Gynecol Cancer. 2008;18:414-20.

3. Buys S, Partridge E, Black A, Johnson C, Lamerato L, Isaacs C, et al. Effect of screening on ovarian cancer mortality: The Prostate, Lung, Colorectal and Ovarian (PLCO) Cancer Screening Randomized Controlled Trial. JAMA. 2011;305:2295-303.

4. Menon U, Gentry-Maharaj A, Hallett R, Ryan A, Burnell M, Sharma A,

et al. Sensitivity and specificity of multimodal and ultrasound screening for ovarian cancer, and stage distribution of detected cancers: results of the prevalence screen of the UK Collaborative Trial of Ovarian Cancer Screening (UKCTOCS). Lancet Oncol. 2009;10:327-40.

5. Woodward E, Sleightholme H, Considine AM, Williamson S, McHugo J, Cruger D. Annual surveillance by CA125 and transvaginal ultrasound for ovarian cancer in both high-risk and population risk women is ineffective. BJOG. 2007;114:1500-9.

6. McAlpine J, Hanley G, Woo M, Tone A, Rozenberg N, Swenerton K, et al. Opportunistic salpingectomy: uptake, risks and complications of a regional initiative for ovarian cancer prevention. Am J Obstet Gynecol. 2014;210:471.e1-11. 
7. Ferreira A, Carvalho M, Rebelo T, Falcão F, Torgal I. A new model of carcinogenesis for ovarian cancer: clinical implications for prophylactic salpingectomy. Acta Obstet Ginecol Port. 2016;10:50-60.

8. Temkin S, Bergstrom J, Samimi G, Minasian L. Ovarian cancer prevention in high risk women. Clin Obstet Gynecol. 2017;60:738-57.

9. Crum C, Drapkin R, Kindelberger D, Medeiros F, Miron A, Lee Y. Lessons from BRCA: the tubal fimbria emerges as an origin for pelvic serous cancer. Clin Med Res. 2007;5:35-44.

10. Tone A, Salvador S, Finlayson S, Tinker A, Kwon J, Lee C, et al. The role of the fallopian tube in ovarian cancer. Clin Adv Hematol Oncol. 2012;10:296-306.

11. Dayem T, Badawy A. Effect of prophylactic salpingectomy on ovarian function in premenopausal women in tertiary referral center. Int J Reprod Contracept Obstet Gynecol. 2017;6:4243-7.

12. Kwon J, McAlpine J, Hanley G, Finlayson S, Cohen T, Miller DM, et al. Costs and benefits of opportunistic salpingectomy as an ovarian cancer prevention strategy. Obstet Gynecol. 2015;125:338-45.

13. ACOG Committee Opinion No. 774: Opportunistic salpingectomy as a strategy for epithelial ovarian cancer prevention. Obstet Gynecol. 2019;133:e279-84

14. Scientific Impact Paper No. 44: The distal fallopian tube as the origin of non-uterine pelvic high-grade serous carcinomas. Obstet Gynaecol. 2015;17:70-1.

15. Powell C, Alabaster A, Simmons S, Garcia C, Martin M, McBrideAllen $\mathrm{S}$, et al. Salpingectomy for sterilization: a change in practice in a large integrated health care system, 2011-2016. Obstet Gynecol. 2017;130:961-7.

16. Garcia C, Moskowitz O, Chisholm C, Duska L, Warren A, Lyons G, et al. Salpingectomy compared with tubal ligation at cesarean delivery: a randomized controlled trial. Obstet Gynecol. 2018;132:29-34.

17. Subramaniam A, Blanchard C, Erickson B, Szychowski J, Leath C, Biggio J, et al. Feasibility of complete salpingectomy compared with standard postpartum tubal ligation at cesarean delivery: a randomized controlled trial. Obstet Gynecol. 2018;132:20-7.

18. Shinar S, Blecher Y, Alpern S, Many A, Ashwal E, Amikam U, et al. Total bilateral salpingectomy versus partial bilateral salpingectomy for permanent sterilization during cesarean delivery. Arch Gynecol Obstet. 2017;295:1185-9.

19. Herman H, Gluck O, Keidar R, Kerner R, Kovo M, Levran D, et al. Ovarian reserve following cesarean-delivery with salpingectomy versus tubal ligation - a randomized trial. Am J Obstet Gynecol. 2017;217:472. e1-6.

20. Chandrasekaran D, Menon U, Evans G, Crawford R, Saridogan E, Jacobs $\mathrm{C}$, et al. Risk reducing salpingectomy and delayed oophorectomy in high risk women: views of cancer geneticists, genetic counsellors and gynaecological oncologists in the UK. Fam Cancer. 2015;14:521-30.

21. Ghezzi F, Cromi A, Siesto G, Bergamini V, Zefiro F, Bolis P. Infectious morbidity after total laparoscopic hysterectomy: does concomitant salpingectomy make a difference? BJOG. 2009;116:589-93.

22. Pacheco A, Costa A, Lanhoso A, Santos A, Rodrigues C, Rebelo C, et al. Consenso sobre contraceção 2020. Lisboa: Sociedade Portuguesa de Ginecologia, Sociedade Portuguesa de Contraceção, Sociedade Portuguesa de Medicina da Reprodução; 2020

23. Pregal A, Gouveia A, Petiz A, Sousa A, Braga A, Felix A, et al. Cancro ginecológico - consensos nacionais 2020. Lisboa: Sociedade Portuguesa de Ginecologia, Sociedade Portuguesa de Oncologia, Sociedade Portuguesa de Radioterapia Oncologia, Sociedade Portuguesa de Anatomia Patológica, Sociedade Portuguesa de Radiologia e Medicina Nuclear, Sociedade Portuguesa de Genética Humana, Ordem dos Médicos; 2020.

24. Jones N, Schulkin J, Urban R, Wright J, Burke W, Hou J, et al. Physicians perspectives and practice patterns toward opportunistic salpingectomy in high- and low-risk women. Cancer Invest. 2016;2;35:51-61.

25. Venturella R, Rocca M, Lico D, Trapasso S, Di Cello A, Gizzo S, et al. Prophylactic bilateral salpingectomy for the prevention of ovarian cancers: what is happening in Italy? Eur J Cancer Prev. 2016;25:410-5.

26. Chene G, de Rochambeau B, Le Bail-Carval K, Beaufils E, Chabert P Mellier G, et al. Current surgical practice of prophylactic and opportunistic salpingectomy in France. Gynecol Obstet Fertil. 2016:44:377-84

27. Potz F, Tomasch G, Polterauer S, Laky R, Marth C, Tamussino K. Incidental (prophylactic) salpingectomy at benign gynecologic surgery and cesarean section: a survey of practice in Austria. Geburtshilfe Frauenheilkd. 2016;76:1325-9.

28. Reade C, Finlayson S, McAlpine J, Tone A, Fung-Kee-Fung M, Ferguson S. Risk-reducing salpingectomy in Canada: a survey of ObstetricianGynaecologists. J Obstet Gynaecol Can. 2013;35:627-34.

29. Kapurubandara S, Qin V, Gurram D, Anpalagan A, Merkur H, Hogg R, et al. Opportunistic bilateral salpingectomy during gynaecological surgery for benign disease: a survey of current Australian practice. Aust N Z J Obstet Gynaecol. 2015;55:606-11.

30. Gill S, Mills B. Physician opinions regarding elective bilateral salpingectomy with hysterectomy and for sterilization. J Minim Invasive Gynecol. 2013;20:517-21.

31. Mikami M, Nagase S, Yamagami W, Ushijma K, Tashiro H, Katabuchi $\mathrm{H}$. Opportunistic bilateral salpingectomy during benign gynecological surgery for ovarian cancer prevention: a survey of Gynecologic Oncology Committee of Japan Society of Obstetrics and Gynecology. J Gynecol Oncol. 2017;28:e52. 